\title{
Neutrons for Materials Research: A Critically Scarce Commodity
}

The symposium on Neutron Scattering for Materials Science, which was held at the 1989 MRS Fall Meeting in Boston, has served to highlight the enormous worldwide increase in the use of neutrons in materials science and technology over the past 15 years. Thermal neutrons, by virtue of their low energy, deep penetration, relatively uniform cross section for light or heavy atoms and ability to sense weak magnetic moments, are superior probes to $x$-rays or electrons for many applications. In fact, an extraordinary variety of new neutron probes have proven indispensable for understanding an increasing variety of materials that underlie our industrial economy, particularly in high technology areas.

Among the most actively pursued applications discussed at the MRS Fall Meeting were the following:

- Structure and magnetism in high temperature superconductors,

- Study of residual stress in critical fabrications (pipeline welds, jet turbine blades),

- Neutron transmutation doping of semiconductors and dopant depth profiling of semiconductors and thin films,

- Polymer structure and diffusion and its relation to other polymer properties, and - The basic structures and structural transformations of metals, ceramics, alloys, and glasses, and the characterization of defects in these materials.

It is troublesome, in a time of such excitement in fields where neutrons make unique contributions, that the United States is suffering a protracted reduction in its ability to provide neutrons for materials research. The Department of Energy, besieged with its own well-publicized problems with weapons production facilities, shut down first the High Flux Isotope Reactor (HFIR) at Oak Ridge National Laboratory (November 1986) and then the High Flux Beam Reactor (HFBR) at Brookhaven
National Laboratory (April 1989) for prolonged safety reviews. This, at a time when the DOE pulsed neutron sources at Argonne and Los Alamos National Laboratories are operating well below capacity due to budget restrictions.

Of course, safety must be the overriding concern in reactor operation, and no one has greater interest in the safe operation of our neutron facilities than the scientists who use them. Nevertheless, the scientific community have belatedly begun to express their conviction that the heightened regard for safety is having unintended, albeit foreseeable, effects that are quite detrimental to the health of the fields that the neutrons serve. For example, an open letter to presidential science adviser Allen Bromley addressing these worries was prepared and signed by nearly 100 participants of the MRS Neutron Scattering Symposium. Their concerns included:

1 . The extended, and unplanned, shutdown of HFIR and HFBR has left the United States without a major research reactor in operation for many months (although the upgraded Neutron Beam Split-Core Reactor at NIST will soon restart to handle a fraction of the demand). Aside from the more immediate and obvious consequences, this situation has compromised the careers of many research students along with the research goals and programs of their faculty advisers. This is occurring when the development of a vigorous and growing neutron scattering community is essential for the future prospects of the new research reactor and neutron scattering facility, the Advanced Neutron Source (ANS), planned at Oak Ridge. Many scientists are now hesitant to continue using neutron scattering techniques and are reluctantly being drawn into research less subject to unpredictable external controls.
2. The new level of safety and security concerns is also greatly increasing the cost of operating these facilities. In addition, the need to provide operating costs has also deflected attention and funds from the research programs and instrumentation upgrades of the HFBR and HFIR. Of equal importance, these pressures are seriously slowing the design of the ANS, which must be developed over the next decade if the United States is to have a state-of-theart neutron capability in the next century. Discontinuous changes in the level of required safety must be met by appropriate infusions of funding to accomplish them. The alternative is further deterioration in our ability to provide the needed capabilities in the neutron sector.

3. Even though the development of pulsed neutron source techniques at Argonne and Los Alamos National Laboratories has been very successful and the requests from the national user community for experimental time have grown steadily, the instrument time available has actually decreased due to cumulative deficiencies in funding extending over a number of years. These funding deficiencies have also limited the development and construction of advanced pulsed-source instrumentation which could significantly enhance the scientific capability of the pulsed sources.

The MRS symposium on Neutron Scattering for Materials Science showed that neutrons are a unique probe for an extraordinarily diverse range of material properties. In order for the U.S. to maintain a leadership role in materials science, it is imperative that the two high flux reactors be restarted as soon as possible and adequate resources be provided to ensure the health of both facilities and the companion pulsed neutron sources. It is also important in setting the nation's science policy that the planning required for the Advanced Neutron Source proceed in a timely way so an adequate supply of neutrons will be available for the next generation of materials scientists.

The authors of this editorial-J.D. Jorgensen (Argonne National Labomatory), S.C. Moss (University of Houston), and S.M. Shapiro (Brookhaven National Laboratory)-served as chairs for the symposium on Neutron Scattering for Materials Science at the 1989 MRS Fall Meeting in Boston.

Editor's Note: The HFIR at Oak Ridge was restarted on January 29 at $11 \mathrm{MW}$ and is already producing research data. However, it will not resume full opemtion $(85 \mathrm{MW})$ until about the second week of April. 